\title{
Laminar Free Convection from A Heated Square Cylinder in Bingham Plastic Fluids
}

\author{
BAIRI LEVI RAKSHITH ${ }^{1}$, DR. PRAGYA MISHRA ${ }^{2}$ \\ ${ }^{1}$ PG Scholar, Mechanical Engineering, Chaithanya Bharathi Institute of Technology, Hyderabad \\ ${ }^{2}$ Assistant professor, Chemical Engineering, Chaithanya Bharathi Institute of Technology, Hyderabad
}

\begin{abstract}
The free convection phenomenon from a heated square cylinder submerged in Bingham Plastic fluids is numerically investigated. The governing equations are solved for a wide range of physical dimensionless parameters, such as Rayleigh number $\left(10^{2} \leq R a \leq 10^{5}\right)$, Prandtl number $(10 \leq \operatorname{Pr} \leq$ 100) and Bingham number $\left(0 \leq B n \leq 10^{7}\right)$. The heat transfer characteristics are investigated in terms of local Nusselt number distribution over the surface of the cylinder surface average Nusselt number. Streamlines, isothermal contours, yielded and unyielded regions are visualized in detail.
\end{abstract}

Indexed Terms- Bingham plastic fluids, square cylinder, Free-convection, Nusselt number, Bingham number, Rayleigh number, Prandtl number

\section{INTRODUCTION}

Bingham plastic fluids are one type of viscoplastic fluid which are characterized by the existence of yield stress or threshold stress. If the applied stress is lower than the yield stress, the fluid behaves like an elastic solid, and if the applied pressure ex- cedes the yield stress, then the fluid behaves as fluid; hence the flow domain in this class of fluids is co spanned by both fluid-like (unyielding region) and solid like (yield region). Heat transfer in the unyielded region occurs mainly by conduction, whereas convection is limited to the yielded part of the system [1-5]. To overcome the fluid's yield stress, a minimum strength of buoyancy induced flow is needed for a given value of fluid yield stress [6]. Most substances of multi-phase nature like foam, suspensions, yoghurt, molten chocolate, cosmetics are examples of Bingham plastic fluids. Heat transfer from a square cylinder has many industrial applications, including the design of novel heat exchanger, cooling of electronic components, thermal treatment of various foodstuffs, fluidized bed drying of fibrous substances. Besides, free convection or natural convection, which al- ways contributes in various proportions to the overall heat transfer, and indeed there are numerous applications in food processing where the only mode of heat transfer is by natural convection. Even in mixed convection applications, natural convection is found to be dominating $[7,8]$.

Over the years, researchers have done significant research, and they investigated the heat transfer characteristics from cylinders of various crosssections, including circular, elliptical, triangular and square in Newtonian and Power-law fluids for forced convection, free convection and mixed connection. K.S.Chang [1] investigated laminar natural convection over a sharp-edged horizontal bar situated in an infinite fluid medium has been investigated numerically and experimentally. Finite-difference solutions to the two-dimensional Naiver-Stokes and energy equations were obtained with a fixed Prandtl number of 0.7 for the two configurations: a hat mate of finite thickness and a square bar. The difficulty associated with the complex physical flow domain was overcome by using body-fitted coordinates. In the numerical study, they found no indication of flow separation for the flat plate, in the range of Rayleigh number $10^{3} \leq \mathrm{Ra} \leq 10^{5}$. For the square bar, however, the boundary layer separated easily at the upper sharp edges for $\mathrm{Ra} \geq 5 \times 10^{5}$ and well-defined twin vortices were identified above the upper horizontal surface. Comparison of the two results, the numerical and the experimental, offered good agreement.

O. Zeitoun and M. Ali. [2] examined over a broad variety of Rayleigh numbers and aspect ratios in ducts with square and rectangular cross sections based on natural convection. Streamlines demonstrate that at a constant Rayleigh number, as the aspect ratio rises, separation and circulation occur above the duct's top 


\section{@ C JUL 2021 | IRE Journals | Volume 5 Issue 1 | ISSN: 2456-8880}

surface, and the isotherms confirm this behavior. Additionally, they discovered that increasing the Rayleigh number reduces the thermal boundary layer along the duct's vertical and bottom surfaces, but not on the top surface, at the centerline, where the plume was rising. Increasing $\mathrm{Ra}$ has a negligible effect on the thermal boundary layer on the top surface, at the centerline, where the plume was rising, and its thickness depends on the solution domain chosen. Additionally, velocity distributions were shown, and velocity profiles showed the circulation and reverse flow detected using streamlines. The total heat transfer from the perimeter of the duct was determined, and the average heat transfer coefficients for different aspect ratios were converted to dimensionless Nusselt values. Zeitoun et al [3] conducted experimental investigations into the average natural convection heat transfer from the isothermal vertical surfaces of a vertical short and slender square cylinder to air and concluded that, below certain Rayleigh numbers, due to curvature effects, the average natural convection heat transfer from the vertical square cylinder's side walls was greater than the data for a vertical flat plate. Kumar Arnab De, Amaresh Dalal [9] examined natural convection around a tilted heated square cylinder kept in an enclosure has been studied in the range of $10^{3} \leq \mathrm{Ra} \leq 10^{6}$. The Navier-Stokes's equation was numerically solved using the finitedifference technique in a non-orthogonal body-fitted coordinate system. The authors provide de- tailed flow and heat transfer characteristics for two distinct thermal boundary situations. The effects of the enclosure shape were evaluated using three different aspect ratios and varying the height of the square cylinder from the bottom. The notion of heat function was used to determine the direction of heat transfer. They discovered a quantifiable difference between uniform wall temperature heating and uniform wall heat flux heating. If the aspect ratio was changed, the flow pattern and thermal stratification are altered. Overall heat transmission was also affected by the aspect ratio. Therefore, they determined that constant wall temperature heating was more efficient than constant wall heat flux heating for the current physical condition in terms of total heat transfer. The location of the heated cylinder was irrelevant.

R.P.Chhabra, C.Sasmal [10] conducted numerical simulations of laminar natural convection heat transfer from a heated long cylinder with a square crosssection immersed in stationary power-law fluids. The governing differential equations (continuity, momentum, and thermal energy) have been solved over wide ranges of the relevant dimension- less parameters, namely, Grashof number $\left(10 \leq \mathrm{Gr} \leq 10^{5}\right)$, Prandtl number $(0.72 \leq \operatorname{Pr} \leq 100)$, and power-law index $(0.3 \leq \mathrm{n} \leq 1.8)$, covering both shear-thinning and shear- thickening type fluid. They investigated the flow's intricate structure in terms of stream- line and isotherm patterns, while analyzing the heat transfer characteristics in terms of the local Nusselt number distribution throughout the cylinder's surface as well as its surface averaged values. In general, the flow stays connected to the surface until the Grashof number is greater than one in shear-thinning fluids ( $n$ $<1)$ than in Newtonian media $(n=1)$. Similarly, shearthinning behavior increases heat transmission when all other fac- tors are equal. Indeed, given the right circumstances, i.e., values of the Grashof number, Prandtl number, and power-law index, it is feasible to increase the rate of heat transfer by up to $100 \%$. Shearthickening fluid behavior has a detrimental effect on heat transfer rate. In the limiting situation of Newtonian fluid behavior $(n=1)$, it was established that for constant values of Rayleigh number, Prandtl number, and Reynolds number.

Nirmalkar, N., Bose, A., and Chabra, R.P. [8] Rayleigh number $10^{2} \leq \mathrm{Ra} \leq 10^{6}$; Prandtl number $30 \leq$ $\operatorname{Pr} \leq 100$; and Bingham number $0 \leq \mathrm{Bn} \leq 10^{4}$ for laminar free convection heat transfer from a horizontal heated cylinder to Bingham plastic fluids across a variety of circumstances was studied. They found that the Nusselt number exhibits a positive relationship with the Rayleigh number, but an inverse relationship with the Bingham and Prandtl number. Heat transfers are greater in shear-thinning fluids than in shearthickening fluids.

Nirmalkar, R.P.Chhabra, and R.J.Poole [11] investigated the momentum and heat transfer properties of a heated square cylinder submerged in a streaming Bingham plastic media. The governing differential equations (continuity, momentum, and thermal energy) have been numerically solved for a variety of circumstances, including the plastic Reynolds number of $0.1 \leq \mathrm{Re} \leq 40$, the Prandtl number 
of $1 \leq \operatorname{Pr} \leq 100$, and the Bing- ham number of $0 \leq \mathrm{Bn}$ $\leq 100$. The flow is anticipated to be symmetrical and constant throughout this range of circumstances. The flow and temperature fields near the cylinder surface are studied in depth using streamline and isotherm profiles. Due to the existence of the yield stress, the flow domain is demarcated into yielded (fluid-like) and unyielded (solid-like) areas as functions of the Reynolds and Bingham numbers. They achieve this goal by doing a thorough analysis of the velocity and shear rate profiles along the centre planes. Additional insights are gained into the pressure and local Nusselt number distributions throughout the cylinder surface, as well as their average values in terms of drag coefficients and mean Nusselt number. They reached an agreement. Additional reliance on Prandtl number exists in the temperature field and heat transfer properties. By and large, all unyielded areas grow as the Bingham number increases. By using an effective viscosity, it is possible to reconcile Nusselt number findings over a broad range of Bingham numbers.

P.Mishra, R.P.Chhabra [12] The heat transfer characteristics of steady flow of Bing- ham plastic fluids over a square cylinder confined in a plane slit have been numerically explored in terms of isotherm contours, local Nusselt number, and average Nusselt number for the following ranges of conditions: Bingham number $0.01 \leq \mathrm{Bn} \leq 100$, Reynolds number $0.1 \leq \operatorname{Re} \leq 40$, Prandtl number, $10 \leq \operatorname{Pr} \leq 100$, and blockage ratio, $(\mathrm{l} / \mathrm{H})=0.2,0.3,0.4$. Additionally, the effect of two distinct thermal boundary conditions, namely constant heat flow condition (CHF) and constant wall temperature (CWT), on the square cylinder's surface has been defined. The focus is on clarifying the impact of blockage ratio on total heat transmission since it affects temperature gradients and adds dissipation at confining walls. Heat transmission occurs in Bingham plastic fluids through two competing mechanisms: convection in fluid-like areas and conduction in solid unyielded regions. As a result, the total heat transmission is complexly controlled by these two competing processes.

It is thus clear that most of the literature is available on natural convection from horizontal square cylinder in Newtonian and power law fluids. Besides, literature on natural convection heat transfer in Bingham plastic fluids is available for circular and elliptical cylinders.
To the best of authors knowledge, there is no prior study on natural convection heat transfer in quiescent Bingham plastic fluids from a heated horizontal square cylinder. Limited results are available on heat transfer in forced convection regimes. This work aims to fill the gap in the current literature. Hence, laminar heat transfers from a heated square cylinder have been numerical studied for over a wide range of parameters such as Rayleigh number $\left(10^{2} \leq \mathrm{Ra} \leq 10^{5}\right)$, Prandtl number $(10 \leq \operatorname{Pr} \leq 100)$ and Bingham number $(0 \leq \mathrm{Bn}$ $\left.\leq 10^{7}\right)$

\section{PROBLEM FORMULATION AND GOVERNING EQUATIONS}

The heat transfer from a horizontal square cylinder with side L (infinitely long in $\mathrm{Z}$ - direction) whose surface is kept at a constant temperature Tw, submerged in a quiescent Bingham plastic fluid at the constant temperature $\mathrm{T}_{o}$, as illustrated in Fig 1 . The density of the fluid will be lowest near the cylinder due to the existing temperature differential

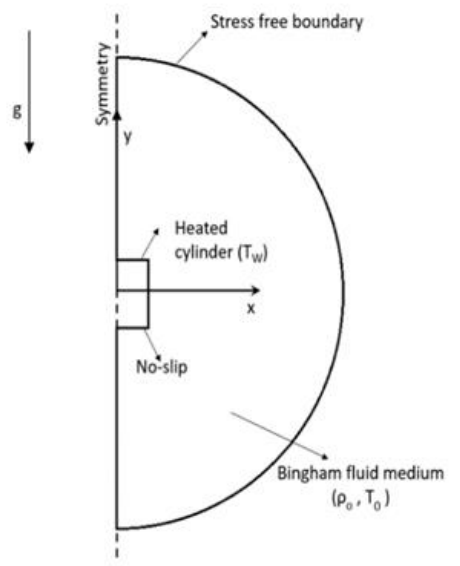

Fig. 1. Schematic diagram of physical model

between the fluid and the square bar, and will progressively rise away from the cylinder, eventually reaching the value corresponding to the distant fluid temperature $T_{0}$. As a result, buoyancy-induced flow occurs, resulting in heat transfer through free (or natural) convection. The viscous dissipation effect is believed to be minimal at this point since the buoyancy generated flow is known to be very modest. Furthermore, the resulting flow is considered to be laminar, steady, two-dimensional, and incompressible (except for the body force term in the $y$ - momentum 
equation) over the range of conditions spanned here, despite the fact that no conclusive information is available in the literature for the transition from one regime to another. Except for the density term in the $y$-momentum equation, the flow is assumed to be symmetric about the vertical centerline of the cylinder and the other thermo-physical properties of the fluid, such as Bingham viscosity, threshold stress, thermal conductivity, and heat capacity, are assumed to be temperature independent. The temperature differential $\mathrm{T}=\mathrm{T}_{w}-\mathrm{T}_{0} \quad$ utilized here is never more than $5 \mathrm{~K}$. For a lower difference between the surface and fluid temperatures, the Boussinesq approximation is employed in the change of fluid density. The Boussinesq approximation is given as: $\rho_{0}-\rho=\rho_{0} \beta\left(T-T_{0}\right)$

Where is the coefficient of thermal expansion at a constant temperature, and it is defined $\beta=-\left.\frac{1}{\rho} \frac{\partial \rho}{\partial T}\right|_{T}$

temperature fields in dimensionless forms.

Continuity equation:

$\frac{\partial U_{x}}{\partial x}+\frac{\partial U_{y}}{\partial y}=0$

$\mathrm{X}$-momentum equation:

$\left(\frac{\partial\left(U_{X} U_{X}\right)}{\partial x}+\frac{\partial\left(U_{X} U_{y}\right)}{\partial y}\right)=-\frac{\partial p}{\partial x}+\sqrt{\frac{\operatorname{Pr}}{R a}}\left(\frac{\partial \tau_{x x}}{\partial x}+\frac{\partial \tau_{y x}}{\partial y}\right)$

Y-momentumequation:

$\left(\frac{\partial\left(U_{y} U_{X}\right)}{\partial x}+\frac{\partial\left(U_{y} U_{y}\right)}{\partial y}\right)=-\frac{\partial p}{\partial y}+\sqrt{\frac{\operatorname{Pr}}{R a}}\left(\frac{\partial \tau_{x y}}{\partial x}+\frac{\partial \tau_{y y}}{\partial y}\right)+T$

Thermal energy equation:

$\frac{\partial\left(U_{x} T\right)}{\partial x}+\frac{\left(\partial U_{y} T\right)}{\partial y}=1 / \sqrt{R a \cdot \operatorname{Pr}}\left(\frac{\partial^{2} T}{\partial x^{2}}+\frac{\partial^{2} T}{\partial y^{2}}\right)$

To make the problem more accessible, in the energy equation, the viscous dissipation is neglected here. for Bingham plastic fluid, the extra stress tensor $\tau$ in dimensionless form is returned as follows:

$$
\begin{aligned}
& \tau=\left(1+\frac{B n}{|\dot{\gamma}|}\right) \dot{\gamma} \text { if }|\tau|>B n \\
& \dot{\gamma}=0 \quad \text { if }|\tau| \leq B n
\end{aligned}
$$

Since the Bingham constitutive relation is nondifferentiable and discontinuous, this form of relation poses difficulties. To avoid these difficulties and provide convergence characteristics and smooth transitions between the yielded and unyielded region, the Herschel-Bulkley model predicts the Bingham plastic model. In the equations as mentioned earlier, length $\mathrm{L}$, velocity $\sqrt{\lg \beta \Delta T}$, viscosity $\mu_{B}$, pressure $\rho_{0} \lg \beta \Delta T$, the rate of strain and stress component are scaled as $\sqrt{g \beta \Delta T / l}, \mu_{B} \sqrt{g \beta \Delta T / l}$. The temperature is non-dimensioalized as $T=\left(T-T_{0}\right) /\left(T_{W}-T_{0}\right)$. The dimensional consideration of these parameters are functions of the three dimensionless numbers, namely, Bingham number (Bn). Rayleigh number (Ra), Prandtl number (Pr). For Bingham plastic fluid, these parameters are defined as follows:

Bingham number:

$B n=\frac{\tau_{0}}{\mu_{B}} \sqrt{\frac{l}{g \beta \Delta T}}$

$\mathrm{Bn} \rightarrow 0$ denotes the limiting case of Newtonian fluid behavior. Besides, $\mathrm{Bn} \rightarrow \infty$ denotes the plastic sold like behavior.

Rayleigh number:

$$
R a=\frac{\rho_{0}^{2} c_{P} g \beta \Delta T l^{3}}{\mu_{B} k}=G r . P r
$$

Grashof number: $G r=\frac{\rho_{0}^{2} g \beta \Delta T l^{3}}{\mu_{B}^{2}}$

Prandtl number: $\quad \operatorname{Pr}=\frac{c_{p} \mu_{B}}{k}$

These definitions are commonly based on these particular preferences of the scaling variables, and truly as will be viewed later, it is manageable to use different scaling variables in the existing case predominant to the modified definitions of Rayleigh and Prandtl numbers, which are corresponded to Bingham number. Hence it is possible to use both definitions. The boundary conditions for the present case are on the square cylinder no-slip condition is given, and the surface temperature is maintained at a constant value of $\mathrm{T}_{\mathrm{W}}=305 \mathrm{~K}$, and the free stream temperature is $\mathrm{T}_{0}=300 \mathrm{~K}$. On the far-field boundary, the radial velocity and its gradient are taken as zero. These conditions are similar to N.Nirmalkar and A. Bose [8] . The flow domain is expected to symmetric about the vertical centerline of the square cylinder. Thus, for this present case, the half domain has been used for the computation, which helps economize the computational effort. The above-mentioned governing equations for these boundary conditions has been calculated numerically in terms of primitive variables (u-V-p-T). The results are further processed to obtain 
streamlines, isotherms contours, size and shape of the yield and un yield regions, the local and average Nusselt number. A Nusselt number is a dimensionless number that gives the heat transfer rate between fluid and the surface of the cylinder. It is defined as $N u_{l}=\frac{h l}{k}$

The average value of the Nusselt number is obtained by surface integration of the local Nusselt number, which is used to estimate the rate of heat transfer between the cylinder and fluid. The objective of this particular problem is to study the heat transfer characteristics and to select the value of numerical parameter like do $\mathrm{Nu}=\int \mathrm{f} \frac{h l}{k}$

The average value of the Nusselt number can be used to estimate the rate of heat transfer between an object and the surrounding fluid. Since the objective of the present study is to study the heat transfer characteristics, and these have been used here only to ensure that the present results are free from the numerical artifacts and to select optimal values of the numerical parameters like domain size, grid. The average Nusselt number is expected to be the function of the Rayleigh number $(\mathrm{Nu})$, Prandtl number $(\mathrm{Pr})$ and the Bingham number $(\mathrm{Bn})$.

\section{NUMERICAL METHODOLOGY}

The above-mentioned governing equations with applied boundary conditions were solved using the ANSYS-FLUENT 2019-R3 version of the finite volume-based solver. The governing equation was solved using a rectangular mesh. Additionally, a twodimensional, stable, laminar viscous, coupled pressure-based solver with a second order unwinding method was utilized to solve the momentum and energy equations' convective components. Pressure velocity coupling was accomplished using the semiimplicit technique for pressure linked equations (SIMPLE) methodology, and the viscosity was specified using a non-Newtonian power-law viscosity model. To approximate the density as a linear function of temperature, the Boussinesq approximation was used. To prevent convergence issues, the solution was initially started with zero velocity everywhere and the same temperature as the distant fluid. For the continuity and $\mathrm{x}$ - and $\mathrm{y}$-components of momentum equations, as well as for the residuals of the energy equation, the relative convergence criteria of $10^{-6}$ are used. Local and average Nusselt numbers had stabilized to four significant digits under these convergence circumstances. The Bingham plastic fluid is calculated using the Herschel-Buckley technique, using $\mathrm{n}=1$ as the power-law index.

- Domain and Grid test:

Without a doubt, the accuracy of the final solution is based not only on the domain's size or the number of grid points, but also on the grid's quality. The domain is defined in this research by the diameter (D) of the cylindrical envelope. The Domain test was performed on values of $\mathrm{Bn}=0, \mathrm{Bn}=10$, and $\mathrm{Bn}=10^{7}$, and the value of D was systematically changed as 200, 300, 400 for $\mathrm{Bn}=0,1000,1200,1300$ for $\mathrm{Bn}=10$, and 1400, 1500, 1600 for $\mathrm{Bn}=10^{7}$, respectively. After careful study, it was determined that domain sizes of $\mathrm{D}=400$ for $\mathrm{Bn}=0$, $\mathrm{D}=1200$ for $\mathrm{Bn}=10$, and $\mathrm{D}=1500$ for $\mathrm{Bn}=10^{7}$ were sufficient to ensure that the findings were free of border effects. The impact of domain independence on the Nusselt number is seen in Table 1.

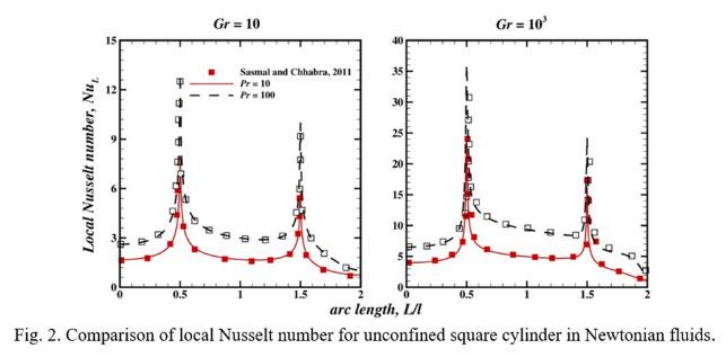

The impact of three computational grids (G1, G2, G3) on these Nusselt number values is shown in Table 2 . Between G2 and G3, there is a difference of less than $1 \%$ in the average Nusselt number values. G2 is sufficient for this research. Hence in summary, all reports reported in this paper are based on the use of G2 with 110000 number of elements in a computational domain depending on Bingham number.

\section{RESULTS AND DISCUSSIONS}

In this particular work, the results have been reported to over a range of $10^{2} \leq \mathrm{Ra} \leq 10^{5}, 10 \leq \operatorname{Pr} \leq 100,0 \leq$ 


\begin{tabular}{|c|c|c|c|c|c|}
\hline \multicolumn{2}{|c|}{ Bn=0 } & \multicolumn{2}{c|}{ Bn=10 } & \multicolumn{2}{c|}{$B n=10^{7}$} \\
\hline $\mathrm{D}$ & $\mathrm{Nu}$ & $\mathrm{D}$ & $\mathrm{Nu}$ & $\mathrm{D}$ & $\mathrm{Nu}$ \\
\hline 200 & 2.1759 & 1000 & 0.3896 & 1400 & 0.2243 \\
\hline 300 & 2.1652 & 1200 & 0.3945 & 1500 & 02221 \\
\hline 400 & 2.1687 & 1300 & 0.3978 & 1600 & 0.2210 \\
\hline
\end{tabular}

Table 1: Domain independence test at $R a=100$ and $\operatorname{Pr}=100$.

\begin{tabular}{|c|c|c|c|c|}
\hline Grid & $\mathrm{Np}$ & $\begin{array}{c}\text { No of } \\
\text { elements }\end{array}$ & $\begin{array}{c}\mathrm{Nu} \\
(\mathrm{Bn}=0)\end{array}$ & $\begin{array}{c}\mathrm{Nu} \\
\left(\mathrm{Bn}=10^{7}\right)\end{array}$ \\
\hline$G 1$ & 280 & 70000 & 8.5896 & 0.2220 \\
\hline$G 2$ & 440 & 110000 & 8.5969 & 0.2222 \\
\hline$G 3$ & 680 & 180000 & 8.6008 & 0.2223 \\
\hline
\end{tabular}

Table 2: Grid independence test at $R a=100$ and $\operatorname{Pr}=100$

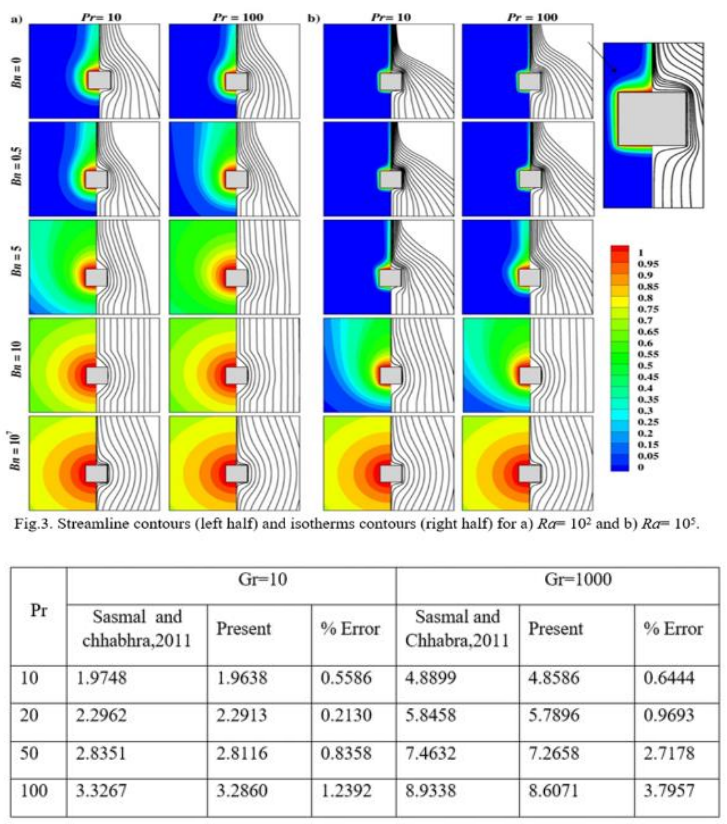

Table 3 Comparison of average Nusselt number in Newtonian fluids for unconfined square cylinder

$\mathrm{Bn} \leq 10^{7}$ The reason to vary these parameters is to describe their influence on the momentum and heat transfer from a square cylinder in a steady free convection regime. Before the detailed presentation and discussion of the obtained results for square cylinder, validation is accomplished here by performing a few benchmark comparisons in the next section with the previous results available in the literature.
- Validation of results:

Extensive numerical results are available in the literature on the Imaging numerical and experimental results are available in literature on the laminar free Convection in Newtonian Fluids from the cylinder. Table 3 is showing a comparison between these predictions in this limit of $\mathrm{B} \rightarrow 0$ for the range of values of Rayleigh number, Prandtl number where as an excellent corresponding value has been seen to the existing between the present values and that of Sasmal and Chabbra, 2011 [10]. In both local and average Nusselt number comparison the value difference is up to $3 \%$ which is in acceptable range as mentioned in the [13] this order of divergence is quite common in such numerical studies because of grid and domain issues at limits of parameters. In both local and average Nusselt number comparison the value difference is up to $3 \%$ which is in acceptable range as mentioned in [8]. This order of divergence is quite common in such numerical studies because of grid and domain issues at limits of parameters

- Streamline and Isotherms:

Due to the lower density of the fluid near the surface of the heated cylinder, the mass of the hot fluid is risen and replaced by the cool fluid from the sides of heated cylinder and that of beneath heated cylinder depending on the value of Rayleigh number. This phenomenon sets up an upward current that directs the formation of plume above the heated cylinder. In Fig 3 The Isotherms and stream lines are visualized based on that phenomenon where the flow is expected to be most vital. Fig 3 shows representative results for Bingham numbers and numbers corresponding to the extreme values of Rayleigh numbers selected here. Such contours also help to por- tray the regions of local cold or that may be relevant in processing temperature sensitive materials. The Scrutiny figure shows that the momentum and thermal boundary layers are progressively thin with the increase of Rayleigh number and increase of number for the fixed values of Bingham number. There will be no yield regions in Bn's limit $\rightarrow \infty$ because as the increase of Bingham number, the yield stress effect suppresses the propensity for fluid deformation and does not allow fluid circulation. Under these conditions, heat transfer occurs by conduction. In the isotherms near the cylinder, the concentric circles appear at $\mathrm{Bn}=10^{7}$ where the limited behavior is approached. Beneath 
these conditions, the yielding is viewed to be constrained to an extremely thin layer adjacent to the surface of the heated cylinder.

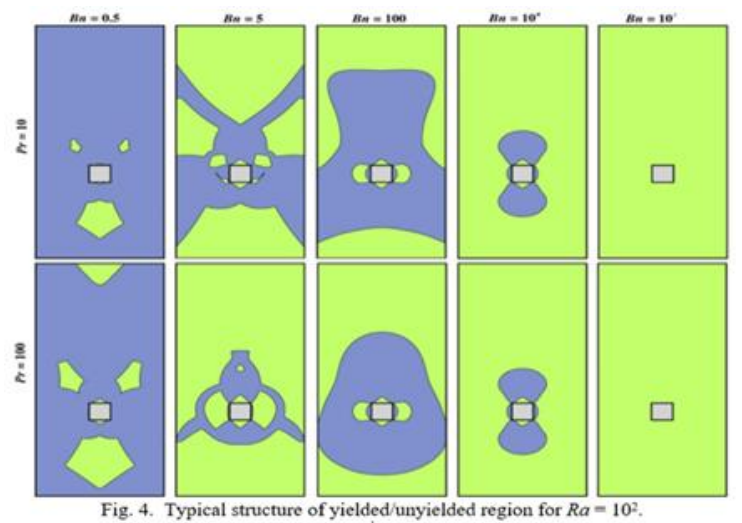

Finally, with the increase in number and number, the velocity and temperature gradients sharpen. Hence, this concludes that these parameters positively depend on the rate of heat transfer from the cylinder to the fluid. Besides, zones grow spatially with the in- crease of the Bingham number, which influences the temperature field and heat transfer rate. Thus, the rate of heat transfer shows a negative dependence on Bingham number. To be sure, the results presented in this section confirm these conjectures

- Morphology of Yielded/ unyielded regions:

As mentioned earlier, the essential characteristic feature which sets fluids to differ from the other Newtonian fluids is the coexistence of fluid-like (yielded) and solid like yielded regions in the fluid domain. The mixing of regions is not so tricky, whereas the heating and the cooling parts tend to be problematic. Scrutiny of the influence of Rayleigh number, number, number on the morphology of the

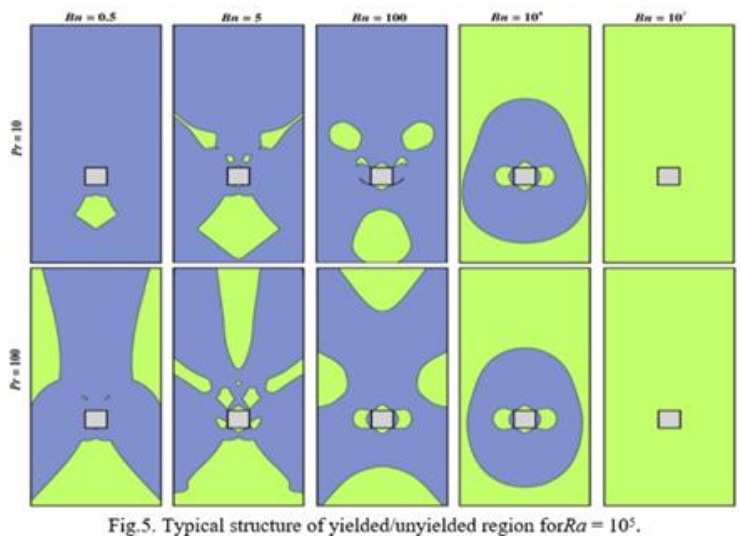

flow fields helps to know the yielded and yielded regions. The deformation rate progressively decreases and becomes zero at the outer boundary where the fluid is at rest. The figure shows the morphology of yielded/regions in the fluid on Bingham number, number, Rayleigh number. After observing the obtained results shown in Fig 4 and Fig 5, for a fixed value of Rayleigh number and number, there are three varieties of regimes fluid-like regions that are obtained depending on the value of Bingham number. Here, the low Bingham number regime is a fluid-like region in the form of a long channel accelerating fluid due to this convective heat transfer dominates under these conditions. In the Intermediate Bingham number regime, fluid-like regions exist near the cylinder region. The material far from the cylinder is still and remains the same. Under this condition, both conduction and convection modes of heat transfer contribute to over- all heat transfer in other portions. High Bingham number regime, there is no fluid as the region is observed. Thus, heat transfer was constrained to the conduction mode. In addition to this, there are three distinct types of the solid region are observed in the intermediate Bingham number regime at the top and bottom as polar cap and almond-like structure to either side of the square cylinder and far outer yielded fluid as can be seen in Fig 4 and Fig 5 at $\mathrm{Pr}=100$ and at $\mathrm{Bn}=10^{4}$.

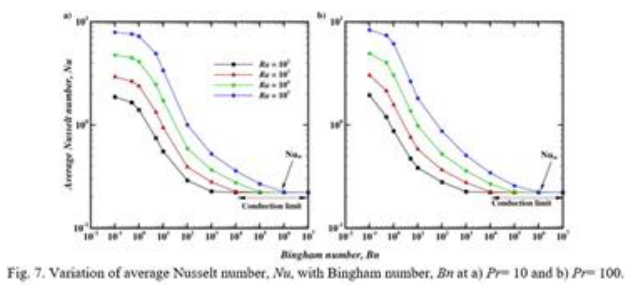


The polar caps are static, and thus heat transfer occurs in the mode of con- duction, whereas in the almondlike structure, the heat transfer is rapid in this part the detailed explanation of local and average number discussed in the following sections.

- Distribution of local number:

The local number is a non-dimensional measure of the heat transfer rate between the cylinder and the fluid. The Bingham plastic fluid behaves like a Newtonian fluid so far that their fluid is characterized by a slope that is the viscosity of the shear stress-share rate plot of the shear stress level more significant than the yield stress. In Newtonian fluids, the local number gradually decreases from its maximum value at the front stagnation point to the rear stagnation point as long as the flow remains attached to the surface of a typical unconfined variation of the local number over the surface of the

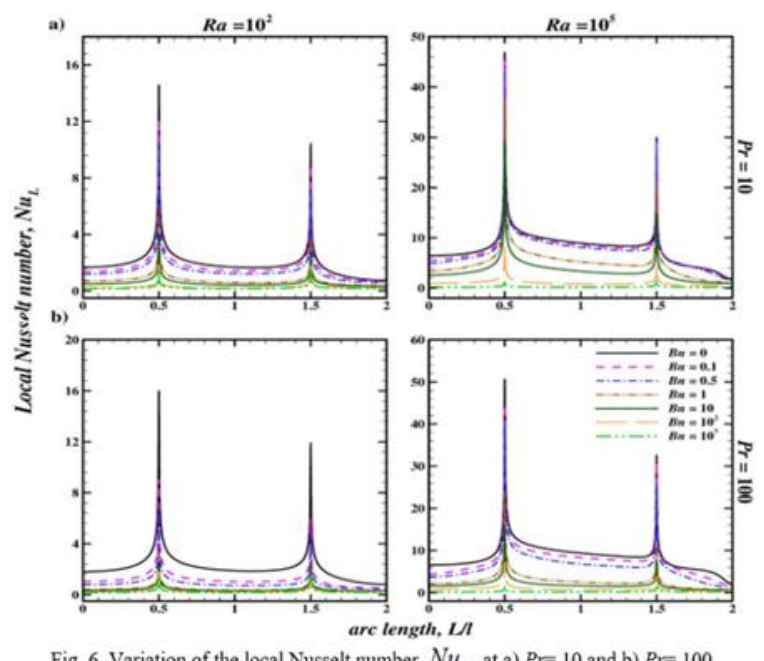

square cylinder is shown in the above Fig 6 , as expected, local number shows the positive dependence on Rayleigh number, number. A detailed examination of these plots suggests that irrespective of the Rayleigh number, the number is maximum at arc lengths of 0.5 and 1.5. From the theoretical standpoint, the temperature gradient at 0.5 and 1.5 is indeterminate, and discontinuity should appear in number because the heat transfer area is zero. In the present work, the mesh is excellent so that no discontinuity appears at that point.
- Average Nusselt number:

As mentioned in the previous section, the average Nusselt number is obtained by sur- face integration of local Nusselt number. After examination of the Fig 7, the Bingham number has a negative dependence on the heat transfer. Fig 7 shows the variation of average Nusselt number with Bingham number $\operatorname{Pr}=10$, Pr $=100$. Dimensional considerations suggest the average Nusselt number be a function of Rayleigh number, Bingham Prandtl number with increase of Bingham number the Average Nusselt number tends to decrease which implies the convective heat transfer was decreasing.

\section{CONCLUSION}

The laminar free convection heat transfer from the heated square cylinder in bingham plastic fluid has been numerically studied using finite volume method for a wide range of parameters such as rayleigh number $(102 \leq \mathrm{ra} \leq 105)$, prandtl number $(10 \leq \mathrm{pr} \leq$ $100)$ and bingham number $(0 \leq b n \leq 107)$. Extensive results on isotherms, streams line, yielded and unyielded regions, local and average nusselt number have been obtained to elucidate the influence of rayleigh, prandtl and bingham numbers on heat transfer. Rayleigh and prandtl number have positive dependence, whereas bingham number has negative dependence on heat transfer.

\section{REFERENCES}

[1] Chang, K. S., Choi, C. J., \& Cho, C. H. (1988). Laminar natural convection heat transfer from sharp-edged horizontal bars with flow separation. International journal of heat and mass transfer, 31(6), 1177-1187.

[2] Zeitoun, O., \& Ali, M. (2006). Numerical investigation of natural convection around isothermal horizontal rectangular ducts. Numerical Heat Transfer, Part A: Applications, 50(2), 189-204.

[3] Turan, O., Chakraborty, N., and Poole, R.J. (2010) Laminar natural convection of Bingham fluids in a square enclosure with differentially heated side walls. Journal of Non-Newtonian Fluid Mechanics, 165 (15-16), 901-913

[4] Turan, O., Chakraborty, N., and Poole, R.J. 
(2012) Laminar Rayleigh-Bénard convection of yield stress fluids in a square enclosure. Journal of Non-Newtonian Fluid Mechanics, 171-172, 83-96,

[5] Turan, O., Poole, R.J., and Chakraborty, N. (2011) Aspect ratio effects in laminar natural convection of Bingham fluids in rectangular enclosures with differentially heated side walls. Journal of Non-Newtonian Fluid Mechanics, 166 (3-4), 208-230

[6] Turan, O., Poole, R.J., and Chakraborty, N. (2012) Influences of boundary conditions on laminar natural convection in rectangular enclosures with differentially heated side walls. International Journal of Heat and Fluid Flow, 33 (1), 131-146,

[7] Nirmalkar, N., Bose, A., and Chhabra, R.P. (2014) Free convection from a heated circular cylinder in Bingham plastic fluids. International Journal of Thermal Sciences, 83, 33-44,

[8] De, A.K. and Dalal, A. (2006) A numerical study of natural convection around a square, horizontal, heated cylinder placed in an enclosure. International Journal of Heat and Mass Transfer, 49 (23-24), 4608-4623

[9] Sasmal, C. and Chhabra, R.P. (2011) Laminar natural convection from a heated square cylinder immersed in power-law liquids. Journal of NonNewtonian Fluid Mechanics, 166(14-15)

[10] Nirmalkar, N., Chhabra, R.P., and Poole, R.J. (2013) Laminar forced convection heat transfer from a heated square cylinder in a Bingham plastic fluid. International Journal of Heat and Mass Transfer, 56 (1-2), 625-639

[11] Mishra, A.K.P., Tiwaria, R.P., and Chhabraa Effect of confinement on the steady flow of Bingham plastic fluids past a heated square cylinder. Heat and Mass Transfer Conference, pp. 2017-2017.

[12] (2012) Fundamentals of heat and mass transfer, P) Ltd, Publisher 